\title{
The Dermatologist Nose Best: Correlation of Nose-Picking Habits and Staphylococcus aureus-Related Dermatologic Disease
}

Matthew LaCour, MD; Kristyna Gleghorn, MD; Janice Wilson, MD

\section{PRACTICE POINTS}

- Staphylococcus aureus colonizes the anterior nares of approximately $20 \%$ to $80 \%$ of humans and can play a large factor in dermatologic disease.

- Staphylococcus aureus decolonization practices for at-risk dermatology patients may overlook the role that nose-picking plays. $\square$ rimitive human habits have withstood the test of time but can pose health risks. Exploring a nasal cavity with a finger might have first occurred shortly after whichever species first developed a nasal opening and a digit able to reach it. Humans have been keen on continuing the long-standing yet taboo habit of nosepicking (rhinotillexis).

Even though nose-picking is stigmatized, anonymous surveys show that almost all adolescents and adults do it. ${ }^{1}$ People are typically unaware of the risks of regular rhinotillexis. Studies exploring the intranasal human microbiome have elicited asymptomatic yet potential disease-causing microbes, including the notorious bacterium Staphylococcus aureus. As many as 30\% of humans are asymptomatically permanently colonized with $S$ aureus in their anterior nares. ${ }^{2}$ These natural reservoirs can be the source of opportunistic infection that increases morbidity, mortality, and overall health care costs.
With the rise of antimicrobial resistance, especially methicillin-resistant $S$ aureus (MRSA), a more direct approach might be necessary to curb nasally sourced cutaneous infection. Since dermatology patients deal with a wide array of skin barrier defects that put them at risk for $S$ aureus-related infection, a medical provider's understanding about the role of nasal colonization and transmission is important. Addressing the awkward question of "Do you pick your nose?" and providing education on the topic might be uncomfortable, but it might be necessary for dermatology patients at risk for $S$ aureus-related cutaneous disease.

Staphylococcus aureus colonizes the anterior nares of $20 \%$ to $80 \%$ of humans; nasal colonization can begin during the first days of life. ${ }^{2}$ The anterior nares are noted as the main reservoir of chronic carriage of $S$ aureus, but carriage can occur at various body sites, including the rectum, vagina, gastrointestinal tract, and axilla, as well as other cutaneous sites. Hands are noted as the main vector of $S$ aureus transmission from source to nose; a positive correlation between nose-picking habits and nasal carriage of $S$ aureus has been noted. ${ }^{2}$

The percentage of $S$ aureus-colonized humans who harbor MRSA is unknown, but it is a topic of concern with the rise of MRSA-related infection. Multisite MRSA carriage increases the risk for nasal MRSA colonization, and nasal MRSA has been noted to be more difficult to decolonize than nonresistant strains. Health care workers carrying $S$ aureus can trigger a potential hospital outbreak

Dr. LaCour is from the Department of Dermatology, Louisiana State University, New Orleans. Drs. Gleghorn and Wilson are from the Department of Dermatology, University of Texas Medical Branch, Galveston.

The authors report no conflict of interest.

Correspondence: Matthew LaCour, MD, LSU Department of Dermatology, 1542 Tulane Ave, New Orleans, LA 70112 (mdlacour04@gmail.com). doi:10.12788/cutis.0100 
of MRSA. Studies have shown that bacterial transmission is increased 40-fold when the nasal host is co-infected by rhinovirus. ${ }^{2}$ Health care workers can be a source of MRSA during outbreaks, but they have not been shown to be more likely to carry MRSA than the general population. ${ }^{2}$ Understanding which patients might be at risk for $S$ aureus-associated disease in dermatology can lead clinicians to consider decolonization strategies.

Nasal colonization has been noted more frequently in patients with predisposing risk factors, including human immunodeficiency virus infection, obesity, diabetes mellitus, granulomatosis with polyangiitis, HLA-DR3 phenotype, skin and soft-tissue infections, atopic dermatitis, impetigo, and recurrent furunculosis. ${ }^{2}$ Staphylococcus aureus is the most frequently noted pathogen in diabetic foot infection. A study found that $36 \%$ of sampled diabetic foot-infection patients also had $S$ aureus isolated from both nares and the foot wound, with $65 \%$ of isolated strains being identical. ${ }^{2}$ Although there are clear data on decolonization of patients prior to heart and orthopedic surgery, more data are needed to determine the benefit of screening and treating nasal carriers in populations with diabetic foot ulcers.

Staphylococcus aureus nasal colonization also has been shown in approximately $60 \%$ of patients with recurrent furunculosis and impetigo. ${ }^{2}$ Although it is clear that there is a correlation between $S$ aureus-related skin infection and nasal colonization, it is unclear what role nosepicking might have in perpetuating these complications.

There are multiple approaches to $S$ aureus decolonization, including intranasal mupirocin, chlorhexidine body wipes, bleach baths, and even oral antibiotics (eg, trimethoprim-sulfamethoxazole, clindamycin). The Infectious Diseases Society of America has published guidelines for treating recurrent MRSA infection, including 5 to 10 days of intranasal mupirocin plus either body decolonization with a daily chlorhexidine wash for 5 to 14 days or a 15 -minute dilute bleach bath twice weekly for 3 months. ${ }^{3,4}$

There are ample meta-analyses and systematic reviews regarding $S$ aureus decolonization and management in patients undergoing dialysis or surgery but limited data when it comes to this topic in dermatology. Those limited studies do show a benefit to decolonization in several diseases, including atopic dermatitis, hand dermatitis, recurrent skin and soft-tissue infections, cutaneous T-cell lymphoma, and surgical infection following Mohs micrographic surgery. ${ }^{4}$ Typically, it also is necessary to treat those who might come in contact with the patient or caregiver; in theory, treating contacts helps reduce the chance that the patient will become recolonized shortly afterward, but the data are limited regarding long-term colonization status following treatment. Contact surfaces, especially cell phones, are noted to be a contributing factor to nares colonization; therefore, it also may be necessary to educate patients on surface-cleaning techniques. ${ }^{5}$ Because there are multiple sources of $S$ aureus that patients can come in contact with after decolonization attempts, a nose-picking habit might play a vital role in recolonization.

Due to rising bacterial resistance to mupirocin and chlorhexidine decolonization strategies, there is a growing need for more effective, long-term decolonization strategies. ${ }^{4}$ These strategies must address patients' environmental exposure and nasal-touching habits. Overcoming the habit of nose-picking might aid $S$ aureus decolonization strategies and thus aid in preventing future antimicrobial resistance.

But are at-risk patients receiving sufficient screening and education on the dangers of a nose-picking habit? Effective strategies to assess these practices and recommend the discontinuation of the habit could have positive effects in maintaining long-term decolonization. Potential euphemistic ways to approach this somewhat taboo topic include questions that elicit information on whether the patient ever touches the inside of his/her nose, washes his/her hands before and after touching the inside of the nose, knows about transfer of bacteria from hand to nose, or understands what decolonization is doing for them. The patient might be inclined to deny such activity, but education on nasal hygiene should be provided regardless, especially in pediatric patients.

Staphylococcus aureus might be a normal human nasal inhabitant, but it can cause a range of problems for dermatologic disease. Although pharmacotherapeutic decolonization strategies can have a positive effect on dermatologic disease, growing antibiotic resistance calls for health care providers to assess patients' nose pickinghabits and educate them on effective ways to prevent finger-to-nose practices.

\section{REFERENCES}

1. Andrade C, Srihari BS. A preliminary survey of rhinotillexomania in an adolescent sample. J Clin Psychiatry. 2001;62:426-431.

2. Sakr A, Brégeon F, Mège J-L, et al. Staphylococcus aureus nasal colonization: an update on mechanisms, epidemiology, risk factors, and subsequent infections. Front Microbiol. 2018;9:2419.

3. Liu C, Bayer A, Cosgrove SE, et al. Clinical practice guidelines by the Infectious Diseases Society of America for the treatment of methicillin-resistant Staphylococcus aureus infections in adults and children: executive summary. Clin Infect Dis. 2011;52:285-292.

4. Kuraitis D, Williams L. Decolonization of Staphylococcus aureus in healthcare: a dermatology perspective. $J$ Healthc Eng. 2018;2018:2382050.

5. Creech CB, Al-Zubeidi DN, Fritz SA. Prevention of recurrent staphylococcal kin infections. Infect Dis Clin North Am. 2015;29:429-464. 